\title{
Anxiety Screening in the Emergency Department
}

Michelle Pinto ${ }^{1}$, Paul Musey, Jr., MD²

${ }^{1}$ Indiana University School of Medicine, ${ }^{2}$ Indiana University School of Medicine, Department of Emergency Medicine

\section{Background and Hypothesis:}

Preliminary research completed in the Indiana University Health Methodist Emergency Department (ED) determined that the prevalence of undetected or unaddressed abnormal anxiety levels in patients with low-risk chest pain was greater than $45 \%$. This subset was noted to have abnormal anxiety symptoms that persisted following visits and increased ED recidivism. We hypothesize that the prevalence of abnormal anxiety in the general ED population will be similar to the subset of patients with low-risk chest pain shown previously.

\section{Methods:}

We enrolled a convenience sample of adult patients with non-psychiatric chief complaints who presented to IUH Methodist and Eskenazi Emergency Departments. Participants were assessed for abnormal anxiety levels using the Generalized Anxiety Disorder 7-item Scale (GAD-7) and the Hospital Anxiety Depression Scale (HADS). Subjects will also complete these assessment tools at 30-days post-enrollment via phone or REDCap survey. Data regarding ED disposition, discharge diagnosis, and ED utilization over the previous 12 months and the 30 days post-enrollment will be collected from the electronic medical record (EMR).

\section{Results:}

Over four weeks, 108 patients were screened and 37 gave informed consent and were enrolled. Preliminary analysis shows that 21 subjects $(56 \%)$ had a GAD-7 score $\geq 10$, indicating abnormal anxiety levels. Full data analysis including comparison of HADS and GAD-7 scores will take place after 50 subjects have been enrolled, completed their 30-day follow-up surveys, and EMR review has taken place.

\section{Conclusion:}

Given data regarding ED visits in patients with low-risk chest pain, identification of anxiety and referral may reduce ED utilization. 